\title{
Enhancing the Accuracy of Radio Tomographic Imaging Using Channel Diversity
}

\author{
Ossi Kaltiokallio \\ Department of Automation and \\ Systems Technology \\ Aalto University School of Electrical Engineering \\ Helsinki, Finland \\ ossi.kaltiokallio@aalto.fi
}

\author{
Maurizio Bocca and Neal Patwari \\ Department of Electrical and \\ Computer Engineering \\ University of Utah \\ Salt Lake City, Utah, USA \\ maurizio.bocca@utah.edu,npatwari@ece.utah.edu
}

\begin{abstract}
Radio tomographic imaging (RTI) is an emerging device-free localization (DFL) technology enabling the localization of people and other objects without requiring them to carry any electronic device. Instead, the RF attenuation field of the deployment area of a wireless network is estimated using the changes in received signal strength (RSS) measured on links of the network. This paper presents the use of channel diversity to improve the localization accuracy of RTI. Two channel selection methods, based on channel packet reception rates (PRRs) and fade levels, are proposed. Experimental evaluations are performed in two different types of environments, and the results show that channel diversity improves localization accuracy by an order of magnitude. People can be located with average error as low as $0.10 \mathrm{~m}$, the lowest DFL location error reported to date. We find that channel fade level is a more important statistic than PRR for RTI channel selection. Using channel diversity, this paper, for the first time, demonstrates that attenuation-based through-wall RTI is possible.
\end{abstract}

Index Terms-device-free localization, radio tomographic imaging, wireless sensor network

\section{INTRODUCTION}

Radio tomographic imaging (RTI) is an emerging technology that enables the device-free localization (DFL) and tracking of people located within the deployment area of a wireless network. In RTI systems, the only sensor used is the radio, which measures the received signal strength (RSS) of signals it receives. Thus we refer to such networks as RF sensor networks [1]. Most importantly, these systems do not require people to carry any device, sensor, or tag to participate in the localization effort. The potential applications of these device-free localization (DFL) systems are many, including ambient assisted living, security systems, rescue operations, and occupational safety systems in industrial areas, among others.

Shadowing-based RTI, introduced in [2] and futher explored in [3], [4], [5], [6], [7], assumes that a person or object attenuates the RSS on a link when they cross through the line between the transmitter and receiver. By measuring the many links that may exist in a wireless network with a mesh topology, an RTI system will measure attenuation on more than one link when a person is in the deployment area, and thus will be able to estimate where the person is located.
However, in indoor environments, the presence of people is not the only factor affecting the propagation of radio signals. Without the presence of a person, the obstructions and objects in the environment affect the multipath propagation environment, that is, the way that the radio signal is shadowed, reflected, diffracted, and scattered [8] on its path from transmitter to receiver. At the receiver, a phasor sum of waves impinging on the antenna determine the received signal strength. This phasor sum may be constructive (waves have same phase) or destructive (waves have opposite phase), resulting in a RSS that is a function of the center frequency and position in space, an effect called multipath fading. When the phasor sum is destructive, the link is in deep fade, and when constructive, the link is in an anti-fade. Person-induced changes in a link's RSS is strongly dependent on fade level [9]. When a person blocks the line between transmitter and receiver, it is the link in an anti-fade that is most strongly and reliably attenuated. Links with other fade levels may see RSS increase or not change, on average, when obstructed. An example multi-channel RSS measurement is shown in Fig. 1, in which a human moves and then stands on the link. On the channel with highest average RSS (\#26), the person's effect is to reduce the RSS; while on the other two channels, the effect is very small (on \#15) or to increase the RSS (on \#11).

In this paper, we show that the localization accuracy of RF networks can be dramatically improved by exploiting multichannel communication among the nodes. By measuring RSS on multiple channels on each link, it is more likely that one of the channels is in an anti-fade, and thus will measure attenuation when a person crosses the link line. Using the RSS measurements from the multiple channels on each link, we propose two channel selection methods, based on the packet reception rate (PRR) and on the channel fade level, to determine which channels' RSS measurements to use in RTI. We analyze how each method affects localization accuracy compared to the use of a single frequency channel. We perform tests in two different indoor environments, one in which links are unobstructed, and another in which the nodes are positioned outside the walls of a cluttered student lounge. The RTI system in the second environment is a see-through walls system like [10]; however, our shadowing-based RTI method 


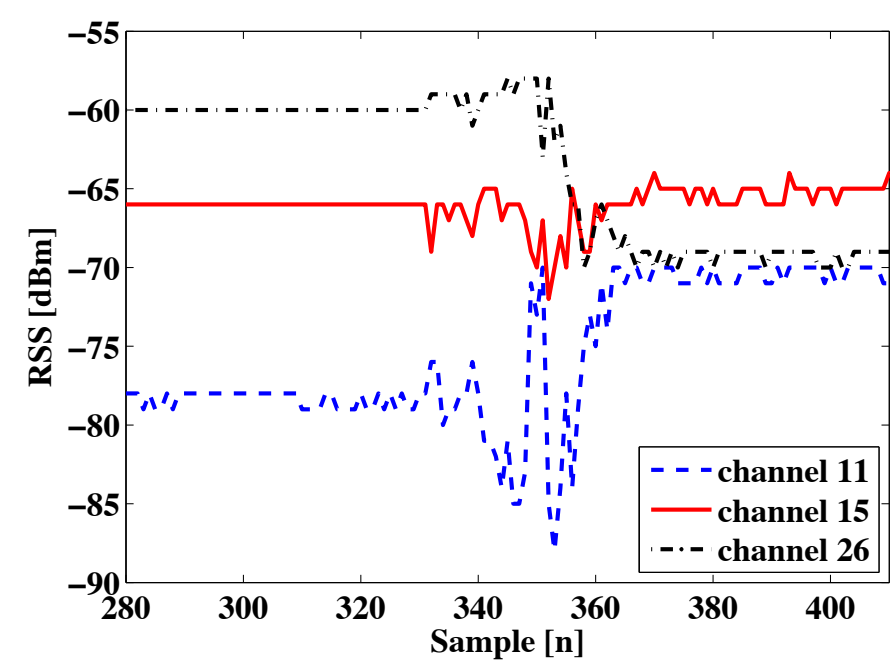

Fig. 1. RSS measurements on three frequency channels on one link when a person moves in between the transmitter and receiver at time $n=355$. The RSS behavior varies considerably among channels.

can locate stationary people while the variance-based method of [10] cannot. The results of this paper are the first to show that shadowing-based RTI can locate people behind walls.

The results also show that the fade level method outperforms the packet reception rate method in terms of localization accuracy. Thus we find that a link's fade level is more important than a link's communication performance in channel selection. To the best of our knowledge, this is the first work demonstrating that RTI's localization accuracy can be consistently improved by channel diversity, even in throughthe-wall scenarios.

\section{A. Related Work}

Several works [3], [4], [7], [10] have already shown that human motion in an area in which wireless nodes are deployed changes the way the radio signal propagates, enabling the localization and tracking of moving individuals from the change of the RF propagation field. Unfortunately, when in a heavily obstructed indoor environment, multipath becomes a serious issue, making the behavior of most of the wireless links unpredictable. In this paper, we use measurements on multiple channels to improve the probability that each link will measure attenuation when a person is located between its transmitter and receiver.

Nodes used in these related works are equipped with lowpower, IEEE 802.15.4-compliant radios operating in the 2.4 $\mathrm{GHz}$ band. The IEEE 802.15.4 standard [11] specifies 16 channels within the $2.4 \mathrm{GHz}$ band. They are numbered from 11 through 26 and are $5 \mathrm{MHz}$ apart, having a $2 \mathrm{MHz}$ bandwidth. The carrier frequency (in $\mathrm{MHz}$ ) of channel $k$ is:

$$
f_{c}=2405+5 \cdot(k-11), \quad k \in[11,26] .
$$

In indoor environments, low-power 802.15.4 networks are often coexisting with more powerful $802.11 \mathrm{~b} / \mathrm{g}$ (WLAN) networks, which also operate in the $2.4 \mathrm{GHz}$ band (although
802.15.4 channels 15, 20, 25 and 26 do not overlap). The communication performance of RF sensor networks can be degraded from both WLAN interference [12] and co-channel or adjacent channel 802.15.4 network interference [13], [14].

To address these interference problems, several multichannel MAC [15], data collection and dissemination protocols [16] specifically designed for sensor networks have been proposed. Channel diversity has also been exploited to improve the efficiency of over-the-air programming [17] and secret key establishment algorithms [18]. All these works assume that, by having pairs or groups of nodes communicating on different frequency channels, the overall performance of the network can achieve improved communication performance and energy efficiency. In contrast, in this work, our purpose for channel diversity is to enhance the localization accuracy.

The rest of the paper is organized as follows. In section II, an extension for the linear model in [3] is derived and a regularized least-squares solution for image reconstruction is described, together with the methods for channel selection and the performance metrics used in the evaluation. Section III discusses the concept of channel fade level and illustrates the effect of fade level on the RSS measurements. Section IV describes the two conducted experiments, presents the results, and discusses the effect of channel diversity on RTI's localization accuracy. Conclusions and future work directions are given in Section $\mathrm{V}$.

\section{Methods}

\section{A. Measurement model}

The objective of an RTI system is to estimate a discretized attenuation field, using the decrease in RSS on links in the network, and locate the people causing the estimated attenuation field. The RSS measured by the receiving node of link $i$ at time $t$ on channel $k$ can be described as:

$$
\begin{aligned}
r_{i, k}(t)= & P_{k}-L_{i, k}-S_{i, k}(t) \\
& +F_{i, k}(t)-v_{i, k}(t), \quad k \in \mathcal{F}
\end{aligned}
$$

where $P_{k}$ is the transmit power, $L_{i, k}$ the large scale path loss, $S_{i, k}(t)$ the shadowing loss, $F_{i, k}(t)$ the fade level (i.e., fading gain), $v_{i, k}(t)$ the measurement noise, and $\mathcal{F}=\{1, \ldots, K\}$ is the set of radio channels used for communication, where $K$ is the number of channels measured. The transmit power $P_{k}$ is a function of the frequency channel $k$ due to differences in antenna impedance matching across a wide frequency band [19]. One of the channel selection methods requires a transmitpower normalized version of the RSS, which we denote $\tilde{r}_{i, k}$ and define as:

$$
\tilde{r}_{i, k}(t)=r_{i, k}(t)-P_{k} .
$$

We refer to the time interval $\left[t_{0}, t_{a}\right]$ as the calibration period, during which measurements are used to calculate the average RSS for each link on each channel. We define the change in RSS from time $t_{a}$ to $t_{b}$ as follows:

$$
\Delta r_{i, k}=r_{i, k}\left(t_{b}\right)-r_{i, k}\left(t_{a}\right)
$$


which has a simplified representation as:

$$
\begin{aligned}
\Delta r_{i, k} & =S_{i, k}\left(t_{b}\right)-S_{i, k}\left(t_{a}\right)+n_{i, k}\left(t_{b}\right)-n_{i, k}\left(t_{a}\right) \\
& =\Delta S_{i, k}+\Delta n_{i, k}
\end{aligned}
$$

where $\Delta n_{i, k}=F_{i, k}\left(t_{b}\right)-F_{i, k}\left(t_{a}\right)+v_{i, k}\left(t_{b}\right)-v_{i, k}\left(t_{a}\right)$ is the sum of the change in fading on channel $k$ and change in measurement noise.

The change in link shadowing is assumed to be a spatial integral of the attenuation field of the monitored area. Some voxels in the discretized attenuation field will affect a particular link's RSS, and some will not. In our discretized model, this corresponds to the fact that each link's change in shadowing is assumed to be a linear combination of the change in voxel attenuations:

$$
\Delta S_{i, k}=\sum_{j=1}^{N} w_{i j} x_{j, k}+\Delta n_{i, k},
$$

where $x_{j, k}$ is the change in attenuation in voxel $j, w_{i j}$ the weight of pixel $j$ for link $i$, and $N$ the number of voxels. The weighting $w_{i j}$ is presented in more detail in Section II-C.

Channel selection is the reduction of a link's multi-channel RSS measurements to a scalar value for each link and each time:

$$
y_{i}=f\left(\Delta r_{i, 1}, \ldots, \Delta r_{i, K}\right)
$$

where $f(\cdot)$ is an arbitrary function which we refer to as the channel selection function. Choices for the selection function are discussed in Section II-B.

When every link of the RF network is considered, the changes in the attenuation field of the monitored area can be now modeled as:

$$
\mathbf{y}=\mathbf{W} \mathbf{x}+\Delta \mathbf{n},
$$

where $\mathbf{y}$ and $\Delta \mathbf{n}$ are $M \times 1$ vectors representing the measured RSS difference and noise of the $M$ links, $\mathrm{x}$ is the $N \times 1$ radio tomographic image to be estimated, and $\mathbf{W}$ is the weight matrix of size $M \times N$ where each column represents a single voxel of the image and each row the weight of each voxel for that particular link. The linear model for shadowing loss is based on the correlated shadowing models in [2], [20], and on the work presented in [3].

\section{B. Choosing the channels}

We consider three different methods for selecting the channels used in RTI, from among those channels measured. In all the previous works, the RSS measurements are collected on a single frequency channel. In this case, the channel selection method is as follows:

- Single channel: All sensors of the network communicate on a single channel defined by the end user as follows: choose a single channel $k$ from the set $\mathcal{F}$. Now, y $=$ $\left[y_{1, k}, \ldots, y_{M, k}\right]^{T}$. This method yields the same results as in [3].

The system presented in this work collects RSS measurements on multiple frequency channels. Thus, we introduce two new methods to select the channels to be used for RTI from among the measured ones. These methods are:

- PRR method: For every link $i \in\{1, \ldots, M\}$, sort the measured packet reception rate (PRR) of channels $k \in$ $\mathcal{F}$. Make a set $\mathcal{A}_{i}$ of size $m$ containing the indices of the $m$ highest channels of link $i$ by PRR and form the measurement $y_{i}$ as given in (9).

- Fade level method: For every link $i \in\{1, \ldots, M\}$, and every channel $k \in \mathcal{F}$, compute the average transmitpower normalized RSS measurements during the calibration period, specifically, $g_{i, k}=\frac{1}{t_{a}} \sum_{t=1}^{t_{a}} \tilde{r}_{i, k}(t)$, where $\tilde{r}_{i, k}(t)$ is given in (3). We use $g_{i, k}$ as a measure of the fade level - if $g_{i, k_{1}}<g_{i, k_{2}}$, the link is in a deeper fade in channel $k_{1}$ than in $k_{2}$. For each link $i$, sort $g_{i, k}$ for all channels $k$. Make a set $\mathcal{A}_{i}$ of size $m$ containing the indices of the $m$ highest channels by fade level $g_{i, k}$. Then, form the measurement vector as in (9).

The measurement vector for the PRR and fade level methods is formed as follows:

$$
\begin{aligned}
y_{i} & =\frac{1}{m} \sum_{k \in \mathcal{A}_{i}}^{m} \Delta r_{i, k}, \\
\mathbf{y} & =\left[y_{1, \mathcal{A}_{1}}, \ldots, y_{M, \mathcal{A}_{M}}\right]^{T} .
\end{aligned}
$$

The PRR method selects the channels so that the communication reliability of the links is maximized, whereas the fade level method selects the channels so that the fade level of the links is maximized. Generally for links $i \neq j$ we have that $\mathcal{A}_{i} \neq \mathcal{A}_{j}$. However, when $m$ equals the total number of channels $K$, then $\mathcal{A}_{i}=\mathcal{A}_{j}=\mathcal{F}$ and the PRR and fade level methods must yield the same result.

\section{Weight model}

The weighting matrix $\mathbf{W}$ represents a spatial model for how each voxel's attenuation impacts each link. We assume that we cannot measure the spatial impact model a priori. Thus, a geometrical model is needed for this relationship. An ellipse model, in which the transmitter and receiver are located at the foci, has been effectively used in [3] and [10]. Voxels $j$ that fall inside link $i$ 's ellipsoid have their weight $w_{i j}$ set to a constant, which is inversely proportional to the square root of the link length. Otherwise, $w_{i j}$ is set to zero. The weighting can be mathematically formulated as follows:

$$
w_{i j}=\frac{1}{\sqrt{d}} \begin{cases}1 & \text { if } d_{i j}^{t}+d_{i j}^{r}<d+\lambda \\ 0 & \text { otherwise }\end{cases}
$$

where $d$ is the link length, $d_{i j}^{t}$ and $d_{i j}^{r}$ are the distances from the center of voxel $j$ to the transmitter and receiver of link $i$, and $\lambda$ is the ellipse excess path length (which tunes the width of the ellipse).

\section{Image formation}

Estimating the image vector $\mathbf{x}$ from the link measurements $\mathbf{y}$ is an ill-posed inverse problem, where the same set of link measurements can lead to multiple different images, i.e., solutions. Therefore, regularization is required. Tikhonov 
regularization [3], [10] and regularized least squares estimators [2], [21] have been used to achieve submeter localization accuracy. Here, we use a regularized least-squares approach:

$$
\begin{gathered}
\hat{\mathbf{x}}=\mathbf{\Pi} \mathbf{y}, \\
\boldsymbol{\Pi}=\left(\mathbf{W}^{T} \mathbf{W}+\sigma_{N}^{2} \mathbf{C}_{x}^{-1}\right)^{-1} \mathbf{W}^{T},
\end{gathered}
$$

where $\sigma_{N}^{2}$ is the noise variance. The a priori covariance matrix $\mathbf{C}_{x}$ is calculated by using an exponential spatial decay:

$$
\left[\mathbf{C}_{x}\right]_{j l}=\sigma_{x}^{2} e^{-d_{j l} / \delta_{c}},
$$

where $d_{j l}$ is the distance from center of voxel $j$ to the center of voxel $l, \sigma_{x}^{2}$ is the variance of voxel attenuation, and $\delta_{c}$ is a correlation parameter that can be used to determine the desired amount of smoothness in the image. The linear transformation $\Pi$ can be calculated beforehand enabling real-time image reconstruction.

\section{E. Performance metrics}

To evaluate the improvement in accuracy provided by channel diversity, two performance metrics are used. The first measure assesses the localization accuracy, while the second measures the reconstructed image error. The location of one person can be estimated by finding the voxel of the RTI image that has the maximum value:

$$
j=\underset{N}{\arg \max } \hat{\mathbf{x}} .
$$

The location estimate is therefore $\hat{z}=z_{j}$. The localization error is calculated as:

$$
e_{l o c}=\left\|\hat{z}-z_{H}\right\|,
$$

where $z_{H}$ is the true human location. Note that estimate in (14) is only capable of locating one person. Multi-target tracking is a difficult problem in RTI and is not a focus of this paper, and thus we do not propose coordinate estimators for the multi-target case. For multi-target RTI, we show qualitatively that performance is improved in Section V, but quantitative performance comparisons are left for future work.

The second performance measure quantifies the localization accuracy and noise of the reconstructed images. For this, the true attenuation field must first be modeled. Some simplifications are necessary because the exact attenuation field is not known. The human body is modeled as a uniformly attenuating cylinder with radius $r_{H}$, as assumed in [3]. When a human is at location $z_{H}$, the true attenuation image $\mathbf{x}_{T}$ can be written as:

$$
\begin{aligned}
x_{T j} & = \begin{cases}1 & \text { if }\left\|z_{j}-z_{H}\right\|<r_{H} \\
0 & \text { otherwise }\end{cases} \\
\mathbf{x}_{T} & =\left[x_{T 1}, \ldots, x_{T N}\right]^{T}
\end{aligned}
$$

where $z_{j}$ is the coordinate of the center of voxel $j$. By normalizing the estimated image $\hat{\mathbf{x}}$ to one, the error of the reconstructed image can be defined as:

$$
\epsilon_{\text {att }}=\max \left\{p, e_{l o c}\right\} \cdot \frac{\left\|\mathbf{x}_{T}-\hat{\mathbf{x}}_{\text {norm }}\right\|^{2}}{N},
$$

where $\hat{\mathbf{x}}_{\text {norm }}$ is the normalized image vector, $N$ is the number of voxels in the image and $p$ is the pixel size and it is used to prevent multiplication by zero.

\section{Channel Fade LeVel}

People moving in the close proximity of a wireless link effect the RSS by shadowing, reflecting, diffracting, or scattering a subset of its multipath components [1], [10]. In an open environment in which the line-of-sight (LoS) path is dominant, a person blocking the LoS of a link will generally attenuate the radio signal. This phenomenon has been succesfully applied to locate humans in [3], [6], [7], [22]. However, when an environment is heavily obstructed and multipath is common, the change in RSS due to human presence becomes unpredictable. The presence of people may cause changes in the RSS even far away from the LoS. On the other hand, the RSS can remain unchanged or even increase [10] when the LoS is obstructed.

The relation between steady-state, narrow-band fading and the temporal fading statistics of the RSS due to human movement is described in [9]. The authors define fade level, a continuum between two extremes, namely a deep fade and an anti-fade, for the fading observed on a wireless link. A link in a deep fade is affected by destructive multipath interference and will most probably experience high variance as the person moves in a wide area near the transmitter and receiver and the line in between them. In addition, a deep fade link's RSS on average increases when the LoS is obstructed. On the contrary, a link in an anti-fade is affected by constructive multipath interference. The RSS of these links varies significantly less due to movement in the area. As their LoS is obstructed, anti-fade links' RSS tends to decrease. Anti-fade links are the most informative for DFL because the area in which a person changes the RSS is small and predictable, largely limited to the straight line between the transmitter and receiver.

Positive values of $F_{i, k}(t)$ in (2) indicate that the wireless link is affected by constructive multipath interference (and thus is in an anti-fade), whereas negative values denote that the link is experiencing destructive multipath interference (and thus is in a deep fade).

Fade level can be quantified, although indirectly, using (2). In (2), fade level is denoted $F_{i, k}$ and can be written as:

$$
F_{i, k}(t)=r_{i, k}(t)-P_{k}+L_{i, k}+S_{i, k}(t)+v_{i, k}(t) .
$$

Here, $r_{i, k}(t)-P_{k}$ is what we call the transmit powernormalized RSS $\tilde{r}_{i, k}(t)$, given in (3). Large scale path loss $L_{i, k}$ and shadowing loss $S_{i, k}(t)$ change very slowly with the center frequency - in this case, our channels are at most $80 \mathrm{MHz}$ apart at $2.4 \mathrm{GHz}$, or at most $3.3 \%$ different. We assume both are effectively not a function of $k$. Thus $F_{i, k}(t)=\tilde{r}_{i, k}(t)+C_{i}+v_{i, k}(t)$. In other words, fade level is a noisy, offset version of transmit power-normalized RSS. Since we can't directly measure $F_{i, k}(t)$, we use $\tilde{r}_{i, k}(t)$ as a substitute for fade level whenever we are considering the $K$ channels on link $i$.

To illustrate the effect of fade level, Fig. 2 plots the RSS measurements on two different channels of a single link 


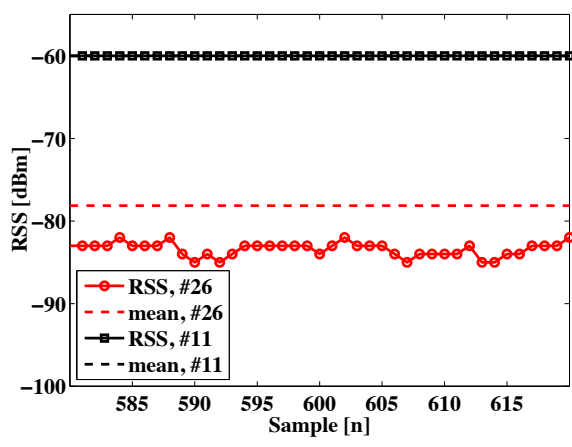

(a) off $\operatorname{LoS}$

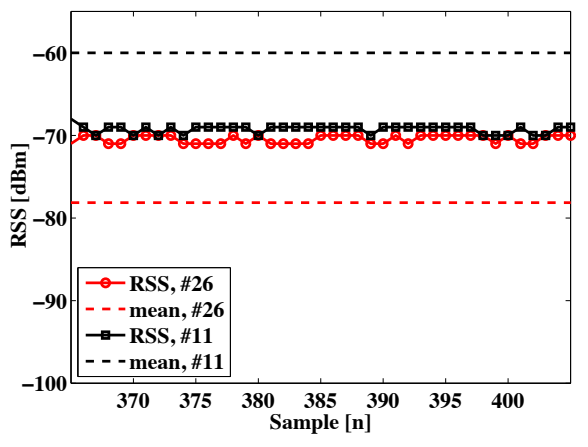

(b) on $\operatorname{LoS}$

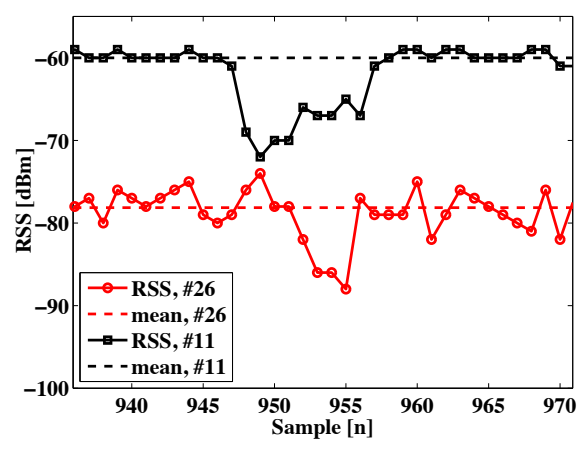

(c) moving along LoS

Fig. 2. Temporal fading of the RSS on two different channels due to human movement, when the line between the TX and RX (a) is not obsructed, and (b) is obstructed. In (c), the person moves in between the nodes, walks along the link line, and then moves away from the nodes.

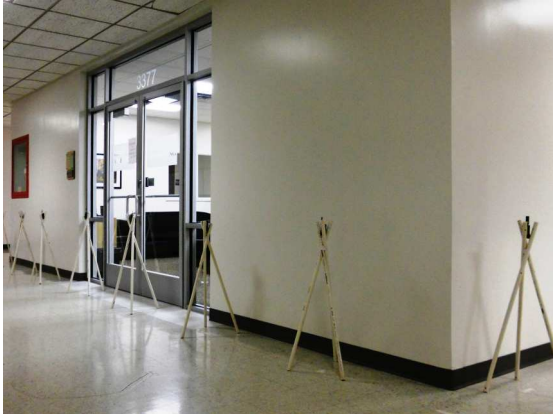

(a) Outside

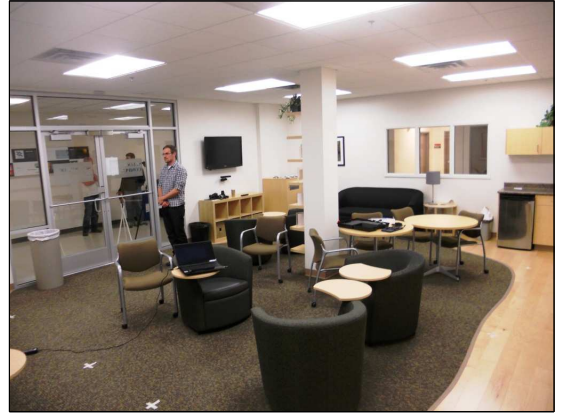

(b) Inside

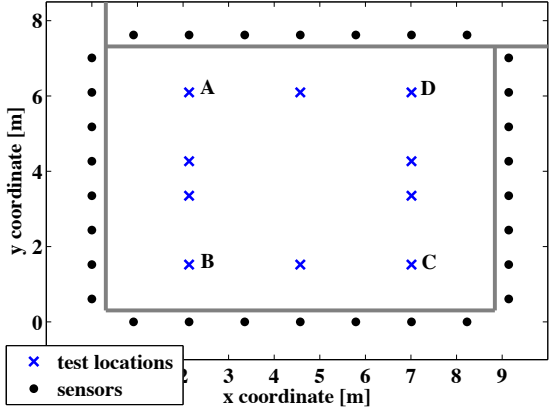

(c) Layout

Fig. 3. The student lounge used in experiment 2 is shown in (a) and (b). In (c), the layout and sensor positions are shown. Experiment 1 uses identical sensor positions, but in an open environment.

(experiment 2), i.e., the LoS link between nodes at $(0.0,1.5)$ and $(9.0,1.5)$, as shown in Fig. 3(c). Note that this link crosses through labeled points B and C in Fig. 3(c). The dashed lines in Fig. 2(a)-(c) show the mean RSS during the calibration period. It can be observed that the fade level difference between the two channels is almost $20 \mathrm{~dB}$. The link can be considered to be in anti-fade on channel 11 and in deep fade on channel 26.

The solid lines graphed in Fig. 2(a) show the RSS when the person is standing at point D, 4.5 meters away from the LoS. On channel 11, the RSS is the same as the one measured during calibration. In contrast, the deep fade channel measures an attenuation in the RSS even though the LoS is not obstructed. In Fig. 2(b), the solid lines show the RSS when the person is standing at point B, i.e. on the LoS. It can be observed that the anti-fade channel experiences attenuation, whereas the deep fade channel experiences an increase in signal strength. In Fig. 3(c), the person moves from point A to point $\mathrm{B}$, reaching the $\operatorname{LoS}$ of the link at sample 948, then walks along the $\operatorname{LoS}$ from point $\mathrm{B}$ to point $\mathrm{C}$, and finally moves off the LoS towards point D at sample 958. In this case, the anti-fade channel measures a small RSS variation until the LoS is obstructed, and a constant attenuation while the person is moving along the LoS. Once the person leaves the LoS, the RSS goes back to the mean value. On the contrary, the deep fade channel starts varying already before the LoS is obstucted, and measures RSS values both higher and lower than the mean while the person is moving along the LoS. Once the person moves away from the LoS, the deep fade channel's RSS continues to vary.

From this example and evidence from the literature [9], [10], we see that links in a deep fade are not reliable indicators of the presence of a person on the line between the transmitter and receiver. However, in obstructed indoor environments, multipath fading is severe and anti-fade links are few. An RTI system that relies on any one channel will have few links accurately measuring person location. On the other hand, when channel diversity is used as we propose, the number of anti-fade links can be considerably increased, consequently improving RTI's localization accuracy.

\section{EXPERIMENTAL RESULTS}

\section{A. Experimental setup}

To evaluate the effect of using channel diversity to RTI, we conduct experiments in two different areas: an open indoor environment (experiment 1) in which all the sensors have LoS communication among each other, and a lounge room (experiment 2) containing furniture and several other objects. Furthermore, in experiment 2 the sensors are placed outside the room, so that the localization is performed through the walls. 


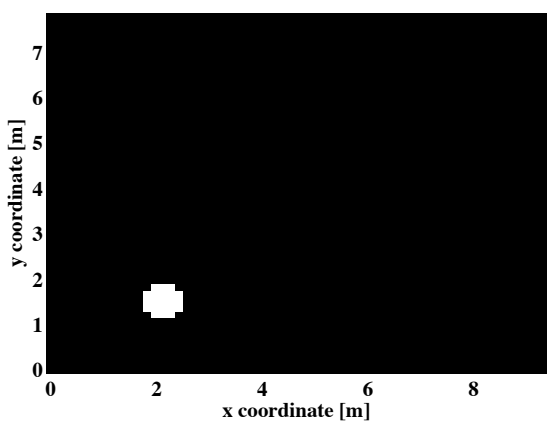

(a) true

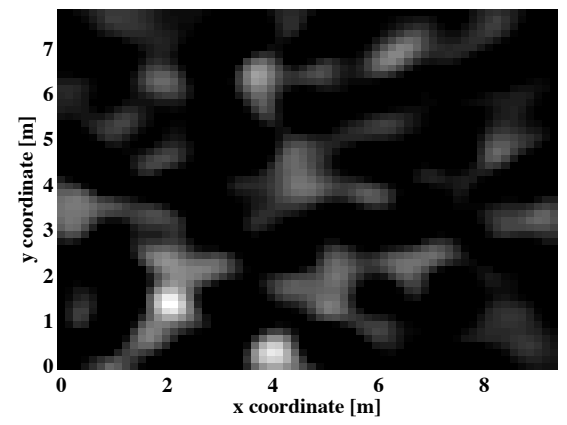

(b) PRR

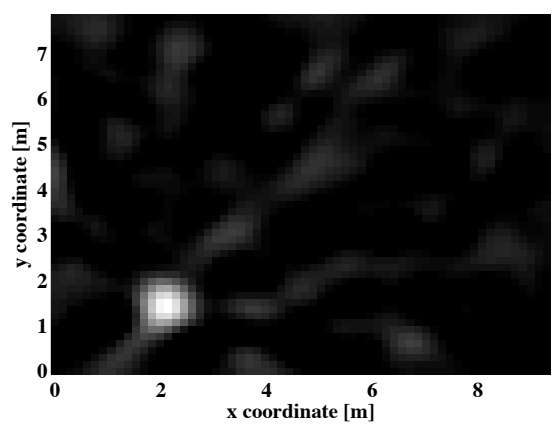

(c) fade level

Fig. 4. Attenuation images of experiment 1 . The person is standing at coordinates $(2.13,1.52)$. The error of the reconstructed image $\epsilon_{a t t}$ is 0.0045 for the PRR method (b) and 0.0008 for the fade level method (c). The number of used channels $m=1$.

TABLE I

PDR OF THE EXPERIMENTS [\%]

\begin{tabular}{cccccc}
\hline \hline Channel & 11 & 15 & 18 & 21 & 26 \\
\hline ex. 1 & 1.1 & 1.4 & 2.5 & 5.7 & 12.3 \\
ex. 2 & 5.3 & 5.2 & 10.8 & 2.5 & 7.8 \\
\hline
\end{tabular}

TABLE II

IMAGE RECONSTRUCTION PARAMETERS USED IN THE EXPERIMENTS

\begin{tabular}{ccc}
\hline \hline Parameter & Value & Description \\
\hline$p$ & 0.1524 & Pixel width [m] \\
$\lambda$ & 0.02 & Excess path length of weighting ellipse [m] \\
$\sigma_{x}$ & 0.2236 & voxels standard deviation [dB] \\
$\sigma_{N}$ & 1 & noise standard deviation [dB] \\
$\delta_{c}$ & 4 & correlation coefficient \\
$r_{H}$ & 0.4 & radius of the human cylinder model [m] \\
$m$ & $1-5$ & number of considered channels \\
\hline
\end{tabular}

In both experiments, 30 sensors are positioned on the perimeter of the monitored area in identical positions, as shown in Fig. 3(c), surrounding a total area of $70 \mathrm{~m}^{2}$. The sensors are placed on podiums at a height of one meter. Figure 3 shows the lounge room used in experiment 2.

In both the experiments, a $60 \mathrm{~s}$ calibration is perfomed in order to measure the RSS of the links in static conditions, i.e., when no people are present in the area. Markers are placed at ten locations, as shown in Fig. 3(c), inside the monitored area. These locations represent the true position of the person during the tests and are used as reference in the subsequent error analysis. The localization error is determined only while the person is standing at one of these ten locations. At each location, the person stands still for $20 \mathrm{~s}$. In this paper, we consider only the localization of stationary people so as to provide an accurate comparison between methods. Although not discussed, the same methods can be applied to track a moving person.

The sensors composing the network are Texas Instruments CC2531 USB dongles set to have $4.5 \mathrm{dBm}$ transmit power [23]. The sensors run a multi-channel token passing protocol, multi-Spin, an extension of Spin [24]. In multi-Spin, the sensors transmit in TDMA fashion with a sequence defined by their ID. When not transmitting, the sensors are in receiving mode. Each packet contains the ID of the transmitting node and the most recent RSS measurements of the packets received from other sensors. If a packet is dropped, the next sensor in the schedule transmits after a back-off time, making the network tolerant to packet drops. At the end of each communication cycle, the sensors switch synchronously to the next frequency channel found in a list pre-defined by the user. On average, the time interval between two consecutive transmissions is $2.9 \mathrm{~ms}$, resulting in a total cycle length of $87 \mathrm{~ms}$. The image reconstruction parameters used in the experiments are listen in Table II.

During the experiments, another network previously deployed in the surrounding rooms and communicating on channel 26 interfered with the communication of our network, which uses channels $11,15,18,21$, and 26 . The packet drop rates (PDRs) of the channels during the two experiments are shown in Table I. As expected, the PDR is on average higher on channel 26 then on the other channels. In addition, the PDR is on average $2 \%$ higher in the cluttered environment.

\section{B. Experiment 1, open environment}

In this section, we quantitatively compare multi-channel and single-channel RTI in an open environment. In Fig. 4, the true attenuation image (a) and the images obtained by applying the PRR (b) and fade level (c) channel ranking methods are shown. The localization errors, calculated as in (15), are 0.15 $\mathrm{m}$ with the PRR method and approximately $0.0 \mathrm{~m}$ with the fade level method. The error of the reconstructed images is $\epsilon_{\text {att }}=0.0045$ with the PRR method and $\epsilon_{a t t}=0.0008$ with the fade level method. These results indicate that the fade level method locates more accurately the person and includes less background noise than the PRR method. Noise in the reconstructed images originates from the RSS measurements of those links that experience changes in the RSS even when a person is not on the LoS. As discussed in Section III, deep fade links are prone to such an unpredictable behavior due to multipath. Thus, the results imply that the fade level method is capable of selecting those channels better suited to achieving high accuracy localization.

The mean position estimates of the PRR and fade level 


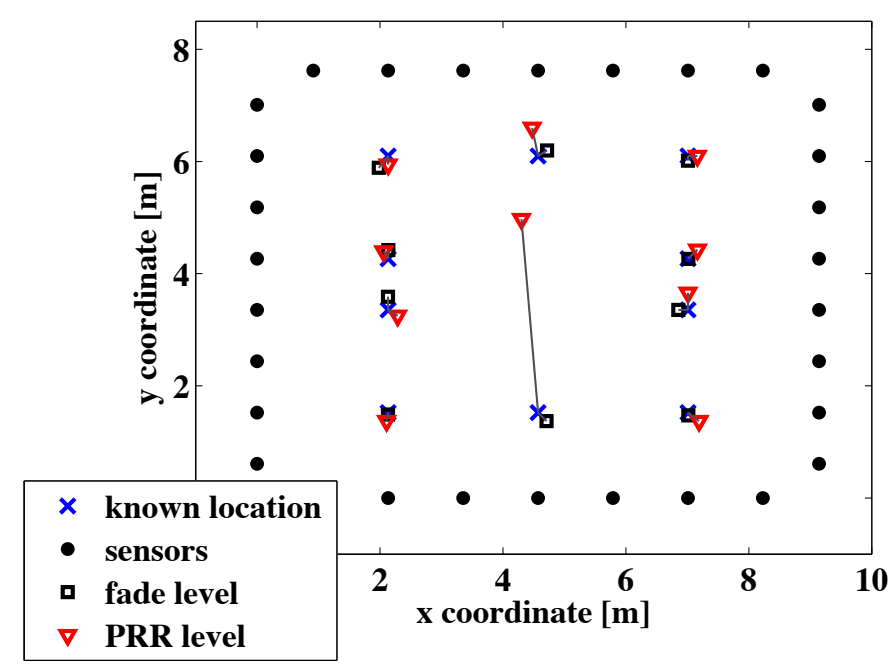

Fig. 5. Localization accuracy of experiment 1. The localization error is $\bar{e}_{l o c}=0.60 \mathrm{~m}$ for the PRR method and $\bar{e}_{l o c}=0.21 \mathrm{~m}$ for the fade level method. The number of used channels is $m=1$.

methods at the ten benchmark positions of the experiment are shown in Fig. 5. The average localization error is $\bar{e}_{l o c}=0.60$ $\mathrm{m}$ for the PRR and $\bar{e}_{l o c}=0.21 \mathrm{~m}$ for the fade level method when the number of used channels is one, i.e., $m=1$. This again indicates that, to obtain accurate position estimates, the link fade level is more important then the link communication performance. The mean localization error for some values of $m$, as reported in Table III, is below the voxel size $p$. In order to reduce further the localization error, the size of the voxel would need to be reduced. However, by reducing it, thus increasing the number of voxels composing the images, the computational complexity of the image reconstruction process increases linearly.

The performance of a system communicating on a single channel is summarized in Table III. The localization accuracy varies considerably between different channels, achieving its best on channel 18, when the localization error is $\bar{e}_{l o c}=1.08$ $\mathrm{m}$ and the mean error of the reconstructed images is $\bar{\epsilon}_{\text {att }}=$ 0.0530 . However, a multi-channel system outperforms the single channel one regardless of the number of selected channels $m$. Moreover, the localization accuracy can be improved by an order of magnitude by applying the fade level method, which achieves its best accuracy when four channels are used $\left(\bar{e}_{l o c}=0.10 \mathrm{~m}\right.$ and $\left.\bar{\epsilon}_{a t t}=0.0018\right)$. The PRR method achieves its best accuracy when all five channels are used $\left(\bar{e}_{l o c}=0.13\right.$ $\mathrm{m}$ and $\bar{\epsilon}_{\text {att }}=0.0032$ ). When $m=K$, the two methods yield the same results.

Fig. 6 shows the distribution of the channels selected by the two methods. The PRR method selects the channels based on their communication performance. For this reason, the distribution follows the PDRs listed in Table I. On the other hand, the distribution of the channels selected by the fade level method is evenly distributed. When a single channel is used, i.e. $m=1$, the PDR for the fade level method is $5.0 \%$, while it is $1.0 \%$ for the PRR method. Since during

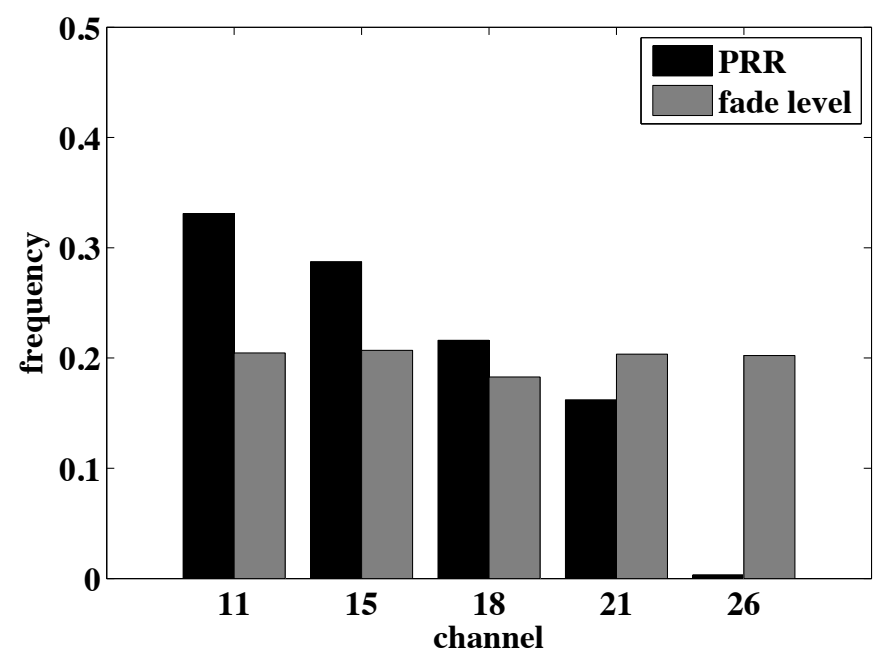

Fig. 6. The distribution of the channels selected by the PRR and fade level methods in experiment 1 . The number of used channels $m=1$.

TABLE III

COMPARISON OF THE DIFFERENT METHODS IN EXPERIMENT 1

\begin{tabular}{c|cc|cc|cc}
\hline \hline & \multicolumn{2}{|c|}{ single } & \multicolumn{2}{c|}{ PRR } & \multicolumn{2}{c}{ fade level } \\
\hline $\begin{array}{c}\text { Channel } \\
k / m\end{array}$ & $\bar{e}_{l o c}$ & $\bar{\epsilon}_{a t t}$ & $\bar{e}_{l o c}$ & $\bar{\epsilon}_{a t t}$ & $\bar{e}_{l o c}$ & $\bar{\epsilon}_{a t t}$ \\
$\left.k / 10^{-3}\right]$ & {$[\mathrm{m}]$} & {$\left[10^{-3}\right]$} & {$[\mathrm{m}]$} & {$\left[10^{-3}\right]$} \\
\hline $11 / 1$ & 1.16 & 50.5 & 0.60 & 23.5 & 0.21 & 5.1 \\
$15 / 2$ & 1.40 & 71.3 & 0.55 & 18.2 & 0.22 & 3.9 \\
$18 / 3$ & 1.08 & 53.0 & 0.22 & 4.7 & 0.14 & 2.4 \\
$21 / 4$ & 2.13 & 97.0 & 0.14 & 3.3 & 0.10 & 1.8 \\
$26 / 5$ & 1.14 & 48.5 & 0.13 & 3.2 & 0.13 & 3.2 \\
\hline
\end{tabular}

the experiment the person is standing still, we can fill the void of the missing RSS measurement of a dropped packet by using the RSS of the last received packet for that link. In this way, we can simulate a system with perfect communication among the nodes. For such a system, the localization accuracy would improve by $16 \%$ for the fade level method and by $5 \%$ for the PRR method. However, when $m$ is increased, the improvement in localization performance provided by a perfect communication flattens out. This indicates that channel diversity allows mitigating the loss of accuracy due to packets drop.

\section{Experiment 2, cluttered environment}

The rich multipath environment of experiment 2 brings forth many difficulties for an attenuation-based RTI system. As can be seen in Fig. 7, the estimated images are significantly corrupted by more noise, and the location estimates are less accurate compared to the open environment of experiment 1 . For example, the localization error in the reconstructed image of Fig. 7 is $e_{l o c}=3.69 \mathrm{~m}$ for the PRR method and $e_{l o c}=0.61$ $\mathrm{m}$ for the fade level method. The single channel method achieves its best accuracy $\left(\bar{e}_{l o c}=1.81 \mathrm{~m}\right)$ on channel 11 . The PRR method achieves its best result when four channels are used $\left(\bar{e}_{l o c}=1.69 \mathrm{~m}\right)$. With the single channel and the PRR methods, the localization accuracy is higher than the one of other proposed systems [10], [21]. However, when these 


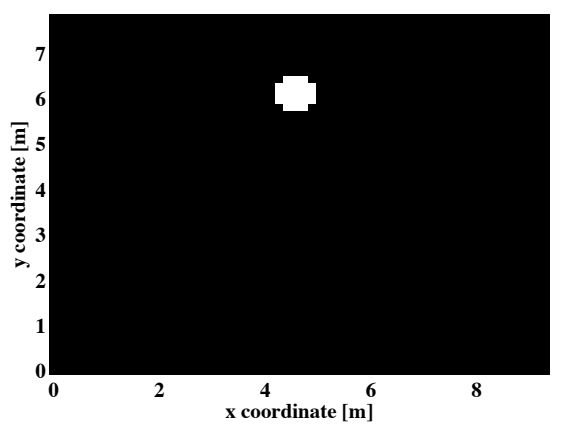

(a) true

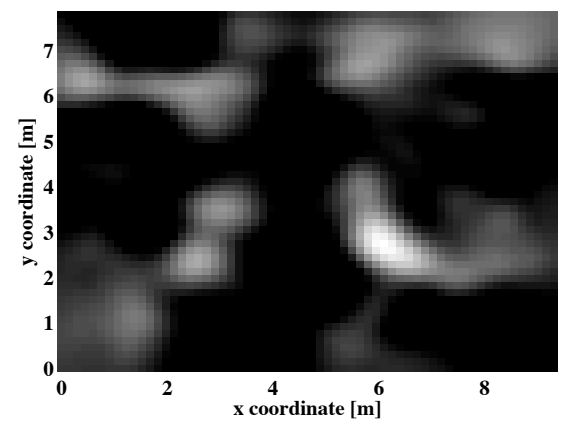

(b) PRR

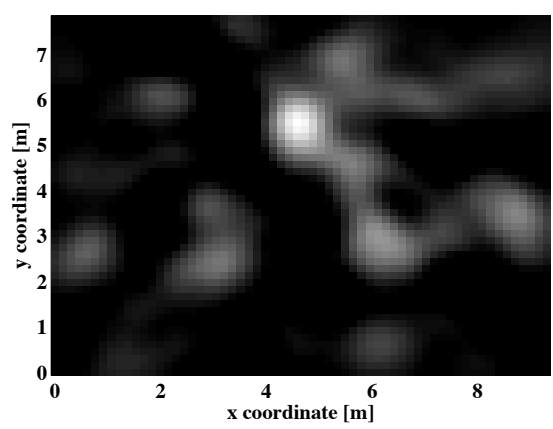

(c) fade level

Fig. 7. Attenuation images of experiment 2. The PRR method (b) does not locate the person correctly. The fade level method (c) locates the person at coordinates $(4.57,5.49)$. The true location (a) of the person is at coordinates $(4.57,6.10)$. The localization error is $0.61 \mathrm{~m}$ for the fade level method and $3.69 \mathrm{~m}$ for the PRR method. The number of used channels is $m=2$.

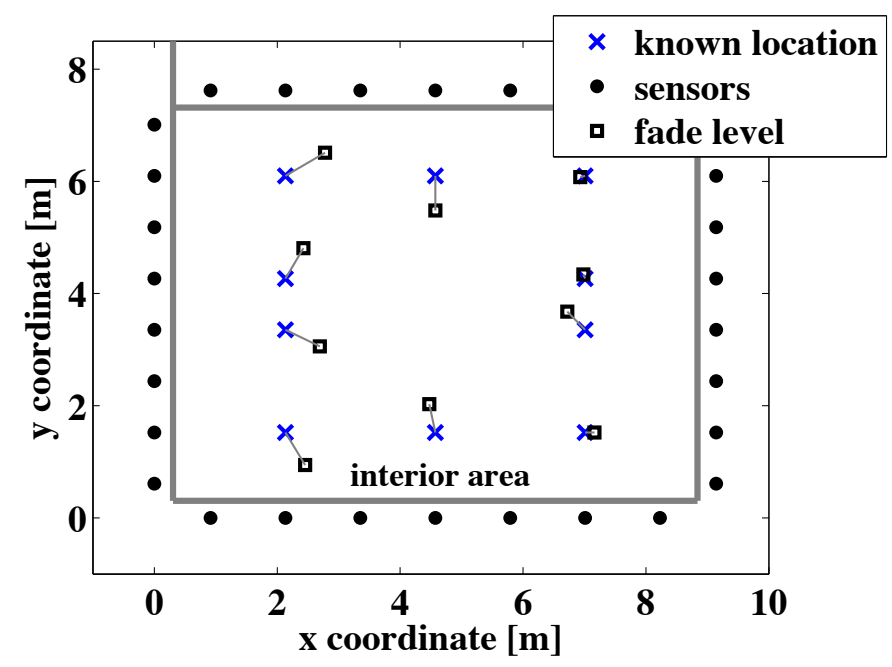

Fig. 8. Localization accuracy of experiment 2 when the fade level method is applied. The number of used channels is $m=3$, resulting in an average localization error of $\bar{e}_{l o c}=0.96 \mathrm{~m}$

two methods are applied, the results agree with what was observed in [10] about the unfeasibility of attenuation-based RTI in highly obstructed areas.

When channel diversity is exploited and the fade level method is applied, the system is capable of achieving submeter localization accuracy also in highly cluttered environments, as shown in Fig. 8. For every link, the method discards the channels that are in deep fade, i.e., prone to experience an increase in signal strength when the LoS is obstructed, and select the ones that are in anti-fade, i.e., experiencing attenuation of the RSS when the LoS is obstructed. The best localization accuracy $\left(\bar{e}_{l o c}=0.96 \mathrm{~m}\right.$ and $\left.\bar{\epsilon}_{a t t}=0.063\right)$ is achieved when $m=3$. The results of the experiments are summarized in Table IV.

In this paper, we exploit channel diversity to enhance the performance of attenuation-based RTI. However, channel diversity can also be used to improve the localization accuracy of other RTI methods, such as variance-based RTI (VRTI)
TABLE IV

COMPARISON OF THE PERFORMANCE OF THE DIFFERENT METHODS IN EXPERIMENT 2

\begin{tabular}{c|cc|cc|cc}
\hline \hline & \multicolumn{2}{|c|}{ single } & \multicolumn{2}{c|}{ PRR } & \multicolumn{2}{c}{ fade level } \\
\hline Channel & $\bar{e}_{l o c}$ & $\bar{\epsilon}_{a t t}$ & $\bar{e}_{l o c}$ & $\bar{\epsilon}_{a t t}$ & $\bar{e}_{l o c}$ & $\bar{\epsilon}_{a t t}$ \\
$k / m$ & {$[\mathrm{~m}]$} & {$\left[10^{-2}\right]$} & {$[\mathrm{m}]$} & {$\left[10^{-2}\right]$} & {$[\mathrm{m}]$} & {$\left[10^{-2}\right]$} \\
\hline $11 / 1$ & 1.81 & 10.2 & 1.90 & 11.3 & 1.55 & 10.8 \\
$15 / 2$ & 2.40 & 13.7 & 2.19 & 14.3 & 1.01 & 6.8 \\
$18 / 3$ & 2.07 & 11.9 & 1.89 & 11.6 & 0.96 & 6.3 \\
$21 / 4$ & 2.68 & 15.9 & 1.69 & 10.8 & 1.21 & 7.7 \\
$26 / 5$ & 2.37 & 13.6 & 1.82 & 10.5 & 1.82 & 10.5 \\
\hline
\end{tabular}

[10]. Since single channel VRTI is capable of localizing people through walls whereas RTI is not, it has to be expected that in such environments multi-channel VRTI will outperform the system presented in this paper. However, this work proves the huge benefits provided by the use of channel diversity. The system described in this paper has been used also in [25], where multi-channel, attenuation-based RTI is applied in a real domestic environment achieving high localization accuracy over extended periods of time.

\section{CONCLUSIONS}

In this paper, the localization accuracy of RTI is improved by means of channel diversity. We introduce a simple, yet effective method to sort the channels used for communication based on their fade level. The accuracy improvement is validated through experimental data collected in two different indoor environments.

The results show that the channels' communication performance is not as important as the temporal fading statistics of the RSS to guarantee a high localization accuracy and image quality. When multiple channels are used, the localization accuracy increases by an order of magnitude compared to a system using a single channel. We demonstrate that channel diversity makes attenuation-based through-wall imaging possible, in contrast to what was reported in the earlier literature.

Considering the improvement in localization accuracy and the reduction in noise, channel diversity represents a powerful technique that should be adopted by the research community active in the area of DFL. The benefits are substantial and will 


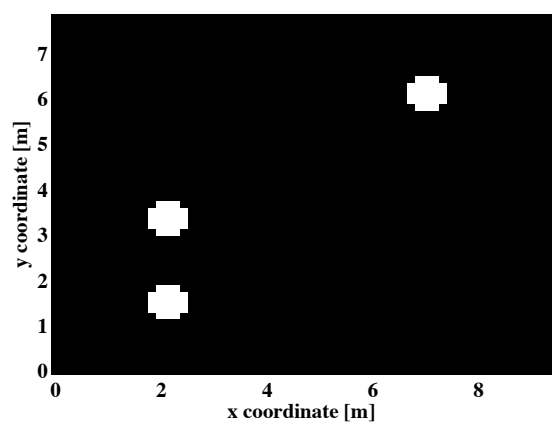

(a) true

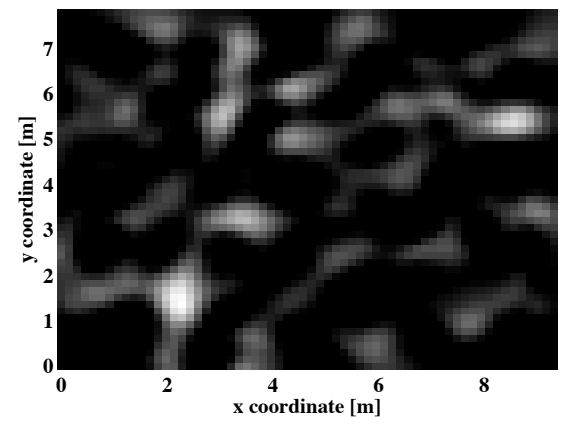

(b) channel 11

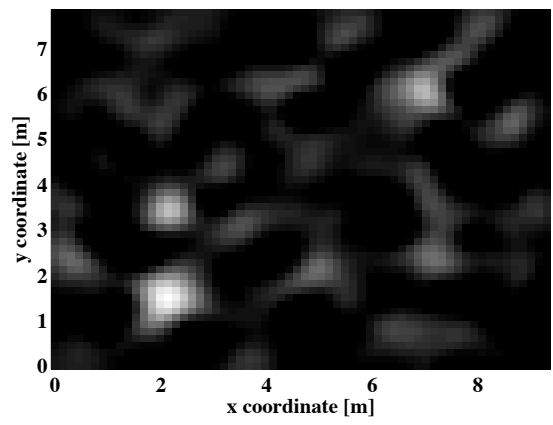

(c) fade level

Fig. 9. The true attenuation field (a) and the reconstructed image using a single channel (b) and the fade level method (c) when three people are monitored in an open environment. The benefit of using channel diversity to locate multiple targets is substantial.

be even more remarkable when more accurate through-wall imaging methods will be developed, especially when multiple target tracking is considered, as shown in Fig. 9.

Future research will investigate more advanced methods for combining the data collected on multiple channels. In addition, since the shadowing and fading caused by objects in highly obstructed areas do not fit well with the LoS weight model, adaptive weight models will be derived.

\section{ACKNOWLEDGMENTS}

This work is funded by the Finnish Funding Agency for Technology and Innovation (TEKES). This material is also based upon work supported by the National Science Foundation under the Early Career Faculty Development (CAREER) Grant No. ECCS-0748206. The authors wish to thank Brad Mager for the help in setting up the experiments.

\section{REFERENCES}

[1] N. Patwari and J. Wilson, "RF sensor networks for device-free localization and tracking," Proceedings of the IEEE, vol. 98, no. 11, pp 1961-1973, Nov. 2010.

[2] N. Patwari and P. Agrawal, "Effects of correlated shadowing: Connectivity, localization, and RF tomography," in IEEE/ACM Int'l Conf. on Information Processing in Sensor Networks (IPSN'08), April 2008, pp. 82-93.

[3] J. Wilson and N. Patwari, "Radio tomographic imaging with wireless networks," IEEE Trans. Mobile Computing, vol. 9, no. 5, pp. 621-632, May 2010, appeared online 8 January 2010.

[4] M. A. Kanso and M. G. Rabbat, "Compressed RF tomography for wireless sensor networks: Centralized and decentralized approaches," in 5th IEEE Intl. Conf. on Distributed Computing in Sensor Systems (DCOSS-09), Marina Del Rey, CA, June 2009.

[5] R. K. Martin, C. Anderson, R. W. Thomas, and A. S. King, "Modelling and analysis of radio tomography," in 2011 4th IEEE International Workshop on Computational Advances in Multi-Sensor Adaptive Processing (CAMSAP), Dec. 2011.

[6] X. Chen, A. Edelstein, Y. Li, M. Coates, M. Rabbat, and M. Aidong, "Sequential Monte Carlo for simultaneous passive device-free tracking and sensor localization using received signal strength measurements," in ACM/IEEE Information Processing in Sensor Networks (IPSN), April 2011.

[7] O. Kaltiokallio and M. Bocca, "Real-time intrusion detection and tracking in indoor environment through distributed rssi processing," in 2011 IEEE 17th Intl. Conf. Embedded and Real-Time Computing Systems and Applications (RTCSA), vol. 1, Aug. 2011, pp. $61-70$.

[8] T. RappaportWireless Communications: Principles and Practice, 2 ed. Upper Saddle River, NJ, USA: Prentice Hall PTR, 2001.
[9] J. Wilson and N. Patwari, "A fade level skew-Laplace signal strength model for device-free localization with wireless networks," IEEE Trans. Mobile Computing, appeared online 12 May 2011.

[10] J. Wilson and N. Patwari, "See through walls: motion tracking using variance-based radio tomography networks," IEEE Trans. Mobile Computing, vol. 10, no. 5, pp. 612-621, May 2011, appeared online 23 September 2010.

[11] IEEE 802.15.4 standard technical specs http://www.ieee802.org/15/pub/TG4Expert.html

[12] K. Srinivasan, P. Dutta, A. Tavakoli, and P. Levis, "Understanding the causes of packet delivery success and failure in dense wireless sensor networks," in SenSys, 2006, pp. 419-420.

[13] Y. Wu, J. A. Stankovic, T. He, and S. Lin, "Realistic and efficient multichannel communications in wireless sensor networks," in INFOCOM, 2008, pp. 1193-1201

[14] G. Xing, M. Sha, J. Huang, G. Zhou, X. Wang, and S. Liu, "Multichannel interference measurement and modeling in low-power wireless networks," in IEEE Real-Time Systems Symposium, 2009, pp. 248-257.

[15] O. Durmaz Incel," "Multi-channel wireless sensor networks: Protocols, design and evaluation, University of Twente, Zutphen, March 2009.

[16] G. Zhou, Y. Wu, T. Yan, T. He, C. Huang, J. A. Stankovic, and T. F. Abdelzaher, "A multifrequency mac specially designed for wireless sensor network applications," ACM Trans. Embedded Comput. Syst., vol. 9, no. 4, 2010 .

[17] W. Xiao and D. Starobinski, "Exploiting multi-channel diversity to speed up over-the-air programming of wireless sensor networks," in Proceedings of the 3rd international conference on Embedded networked sensor systems, ser. SenSys '05. New York, NY, USA: ACM, 2005, pp. 292-293 http://doi.acm.org/10.1145/1098918.1098960

[18] B. Zan and M. Gruteser, "Random channel hopping schemes for key agreement in wireless networks," in PIMRC, 2009, pp. 2886-2890.

[19] Texas Instruments. Small Size $2.4 \mathrm{GHz}$ PCB antenna www.ti.com/lit/an/swra117d/swra117d.pdf

[20] P. Agrawal and N. Patwari, "Correlated link shadow fading in multi-hop wireless networks," IEEE Trans. Wireless Commun., vol. 8, no. 8, pp. 4024-4036, Aug. 2009.

[21] Y. Zhao and N. Patwari, "Noise reduction for variance-based device-free localization and tracking," in 8th IEEE Conference on Sensor, Mesh and Ad Hoc Communications and Networks (SECON'11), June 2011.

[22] M. A. Kanso, "Compressed RF tomography: Centralized and decentralized methods," Master's thesis, McGill University, 2009.

[23] Texas Instruments. A USB-enabled system-on-chip solution for $2.4 \mathrm{GHz}$ IEEE 802.15.4 and ZigBee applications http://www.ti.com/lit/ds/symlink/cc2531.pdf

[24] J. Wilson. SPIN: TinyOS code for RSS collection http://span.ece.utah.edu/spin

[25] O. Kaltiokallio, M. Bocca, and N. Patwari, "Follow @grandma: Longterm device-free localization for residential monitoring," in the 7th IEEE International Workshop on Practical Issues in Building Sensor Network Applications, October 2012, to appear. 